\title{
Irrungen und Wirrungen der Forschung in der hausärztlichen Praxis
}

Oft beklagen sich praktizierende Ärztinnen und Ärzte, «gute» Journals würden häufig praxisfremde Studien publizieren. Im Anschluss an die Veröffentlichung einer grossen englischen Studie, die für eine prophylaktische Gabe von Vitamin D3 bei zu Hause lebenden Senioren günstige Effekte beschrieb, beschlossen wir im lokalen Qualitätszirkel Arbon und Umgebung, eine Studie in Alters- und Pflegeheimen zu lancieren, um die Ergebnisse der englischen Studie zu überprüfen und ihre Übertragbarkeit auf Alterspflegeheimpatienten zu evaluieren.

In einer ersten Studie wollten wir bei geriatrischen Patienten des lokalen Alterspflegeheimes eine Blutentnahme durchführen, um die Prävalenz des Vitamin-D-Mangels in dieser Bevölkerungsgruppe $\mathrm{zu}$ bestimmen. In einer zweiten multizentrischen placebokontrollierten Studie wollten wir die Effekte einer Vitamin-D-Supplementation versus Placebo bei je 1000 Personen untersuchen. Von der Streuli AG in Uznach hätten wir das Studienmedikament und das Placebo gratis erhalten. Die Alterspflegeheime und die Studienleitung hätten ehrenamtlich gearbeitet. So weit, so gut - wir wollten mit der Studie starten.

Nun kamen die Probleme! Wir legten das Studienprotokoll dem ethischen Komitee des Kantons Thurgau vor. Das Protokoll wurde für gut, der Versuch für wissenschaftlich wertvoll befunden. Da es sich aber um eine multizentrische Studie handelte, hätten wir für das Protokoll eine Haftpflichtversicherung abschliessen müssen, wie es das Bundesgesetz über Arzneimittel und Medizinalprodukte, welches seit Januar 2002 in Kraft ist, vorschreibt. Die Berufshaftpflichtversicherung (AXA) des Schreibenden nahm von einer Versicherung der Studie Abstand, da sie keine Studien versichere. Die Zürich und die Winterthur Versicherung offerierten eine Haftpflichtversicherung für Fr. 35.- pro Teilnehmer oder Fr. 70000.- insgesamt. Diesen Betrag wollten wir nicht aus der eigenen Tasche bezahlen und Sponsoren fehlten!
Unsere Studie ist bereits an der Frage der Haftpflichtversicherung gescheitert, bevor sie überhaupt begonnen hat! Welche Lehren können wir daraus ziehen? Die finanziellen Hürden für wissenschaftliche Versuche am Menschen sind sehr hoch, so hoch, dass sich nur grosse Pharmafirmen solche Versuche leisten können. Allerdings führen diese nur Versuche mit potenziell verkäuflichen und patentierbaren bzw. patentierten Substanzen durch, was soweit auch verständlich ist. Studien mit Medikamenten, deren Patentschutz abgelaufen ist oder bei denen keine wirtschaftlichen Interessen bestehen (orphan drugs), sind dagegen wirtschaftlich nicht interessant und werden nicht durchgeführt. Wenn sich ein Praktiker wissenschaftlich betätigen will, muss er sich an pharmaindustriebasierten Studien beteiligen oder sich mit interessanten Fallbeschreibungen zufriedengeben. Klinisch-interventionelle Studien bleiben hingegen der Industrie oder gegebenenfalls den Universitätsspitälern vorbehalten.

Kann an dieser Situation etwas geändert werden? Hausärzte und -ärztinnen könnten sich beispielsweise vernetzen, um gemeinsam aufzuzeigen, welche Studien überhaupt für die Praxis interessant sein könnten und durchgeführt werden sollten. Weiter könnten die wirtschaftlichen Rahmenbedingungen geschaffen werden, welche die nichtkommerzorientierte Erprobung von patentfreien Substanzen überhaupt erlauben, z. B. durch die Übernahme allfälliger Haftpflichtprämien. Auch sollten vom Kollegium für Hausarztmedizin, von den Fachgesellschaften der Grundversorger (Allgemein- und Innere Medizin sowie Pädiatrie) und von den Lehrstühlen für Allgemeinmedizin Know-how für wissenschaftlich interessierte Praktiker(innen) zur Verfügung gestellt werden.

Wenn diese Anregungen auf fruchtbaren Boden fielen, wären die Arbeiten um unsere Vitamin-D-Studie zumindest nicht ganz vergebens gewesen.

Dr. med. Markus Gnädinger, Steinach 